\title{
Roles of central renin-angiotensin system and afferent renal nerve in the control of systemic hemodynamics in rats
}

\author{
Yoshihide Fujisawa ${ }^{1}$, Yukiko Nagai ${ }^{1}$, Bai Lei ${ }^{2}$, Daisuke Nakano $^{2}$, Toshiki Fukui ${ }^{3}$, Hirofumi Hitomi ${ }^{2}$, \\ Hirohito Mori ${ }^{4}$, Tsutomu Masaki ${ }^{4}$ and Akira Nishiyama ${ }^{2}$
}

Afferent renal nerves (ARNs) convey signals generated by physiological changes in the kidney to the central nervous system. The aim of this study was to determine whether ARNs contribute to cardiovascular regulation through central renin-angiotensin system (RAS)-dependent pathways. Blood pressure and renal sympathetic nerve activity (RSNA) were monitored during elevations in pelvic pressure in anesthetized Wistar-Kyoto Izm (WKY) rats and spontaneously hypertensive Izm rats (SHRs). In both groups of rats, blood pressure and RSNA were significantly increased in response to elevations in renal pelvic pressure in a pressure-dependent fashion, which were prevented by renal denervation. Injection of an angiotensin II type I receptor blocker $(\mathrm{CV}-11974,10 \mu \mathrm{g})$ into the intracerebroventricular region significantly suppressed the vasopressor and sympathoexcitatory responses to the increases in pelvic pressure in both WKY rats and SHRs, although these inhibitory effects of CV-11974 in SHRs appeared to be weaker than in WKY rats. These results indicate that signals transmitted by ARNs have an important role in the control of systemic hemodynamics through regulating central RAS-mediated changes in sympathetic nerve activity. Hypertension Research (2011) 34, 1228-1232; doi:10.1038/hr.2011.115; published online 28 July 2011

Keywords: blood pressure; CV-11974; pelvic pressure; renal sympathetic nerve activity

\section{INTRODUCTION}

There is substantial evidence that afferent renal nerves (ARNs) convey signals generated by physiological changes in the kidney to the central nervous system (CNS). ${ }^{1}$ It has also been shown that stimulation of ARNs leads to an increase in systemic sympathetic activity through central sympathetic regulation. For example, electrical stimulation of ARNs elicits an increase in blood pressure via sympathoexcitation and enhancement of vasopressin release. ${ }^{2}$ Furthermore, stimulation of the kidney with intrarenal infusion of specific chemical agents elevates ARN activity and results in an increase in blood pressure, which can be attenuated by anesthesia and prevented by renal denervation. ${ }^{3,4}$ Ye et $a l^{5}$ showed that renal injury induced by phenol resulted in permanent hypertension, which was associated with an increase in norepinephrine release from the posterior hypothalamic nuclei. They also showed that the above phenol-induced changes were completely prevented by renal denervation. Early clinical studies have shown that renal denervation after bilateral nephrectomy ameliorated muscle sympathetic overactivity and lowered blood pressure in patients with chronic kidney disease. ${ }^{6}$ More recently, renal denervation by mechanical nerve ablation for 12 months significantly lowered muscle sympathetic nerve activity and blood pressure in severely hypertensive patients who were not well controlled by anti-hypertensive agents. ${ }^{7}$ These data indicate that ARNs have an important role in the pathogenesis of hypertension through enhancement of systemic sympathetic activity by transmitting information from the diseased kidney to the CNS.

Intracerebroventricular (ICV) administration of angiotensin II (Ang II) has been shown to increase renal sympathetic nerve activity (RSNA) and blood pressure. ${ }^{8}$ Microinjection of Ang II into the paraventricular nucleus enhanced the reflex excitation of RSNA in response to the cardiac afferent stimulation. ${ }^{9}$ In contrast, administration of an AT1 receptor antagonist into the rostral ventrolateral medulla decreased blood pressure in hypertensive rats. ${ }^{10}$ These data suggest that the CNS renin-angiotensin system (RAS) has an important role in controlling systemic sympathetic activity through its excitatory influence on sympathetic regulation in the brain. However, involvement of the CNS RAS in the ARN-mediated regulation of systemic sympathetic activity and hemodynamics is not well understood.

The objective of the present study was to test the hypothesis that ARNs have an important role in the control of systemic autonomic and hemodynamic systems through regulating the CNS

\footnotetext{
${ }^{1}$ Life Science Research Center, Faculty of Medicine, Kagawa University, Ikenobe, Miki-cho, Kita-gun, Japan; ${ }^{2}$ Department of Pharmacology, Faculty of Medicine, Kagawa University, Ikenobe, Miki-cho, Kita-gun, Japan; ${ }^{3}$ Center for Preventive Medical Treatment, NTT West Takamatsu Hospital, Kanko-cho, Takamatsu, Japan and ${ }^{4}$ Department of Gastroenterology and Neurology, Faculty of Medicine, Kagawa University, Ikenobe, Miki-cho, Kita-gun, Japan

Correspondence: Dr Y Fujisawa, Life Science Research Center, Faculty of Medicine, Kagawa University, 1750-1 Ikenobe, Miki-cho, Kita-gun, Kagawa 761-0701, Japan. E-mail: recfuji@kms.ac.jp
}

Received 22 February 2011; revised 25 April 2011; accepted 16 May 2011; published online 28 July 2011 
RAS-dependent pathways. To test this hypothesis, we examined the effects of ICV administration of an AT1 receptor antagonist on responses of RSNA and blood pressure to ARN activation, induced by increasing renal pelvic pressure in rats. It has been considered that the ARN activity is increased by stimulation of the sensory receptors in the kidney. And as the sensory receptors near the pelvic wall are distinctively sensitized to changes in the pelvic pressure ${ }^{11-13}$ or backflow of the urine into the pelvis, ${ }^{14}$ elevation of the pelvic pressure has been a useful tool to activate ARNs. ${ }^{11-13}$

\section{METHODS}

Male Wistar-Kyoto Izm (WKY) rats and spontaneously hypertensive Izm rats (SHRs) weighing 290-350 g (SLC Japan, Hamamatsu, Japan) were used. Rats were housed in separate cages in a temperature-controlled room with a 12 -h light and dark cycle, and were fed a standard laboratory diet and water ad libitum. All surgical and experimental procedures were approved by the Animal Care and Use Committee, Kagawa University, and conformed to the Guidelines for Animal Experimentation of Kagawa University.

\section{Implantation of arterial, venous and ureteral catheters and the renal nerve electrode}

Under sodium pentobarbital anesthesia (50 $\mathrm{mg} \mathrm{kg}^{-1}$, intraperitoneally), a polyethylene catheter (PE-60) was inserted into the abdominal aorta via the right femoral artery for measurement of blood pressure. Another catheter (PE-50) was inserted into the inferior vena cava via the right femoral vein for administration of saline $\left(2 \mathrm{ml} \mathrm{h}^{-1}\right)$. Supplemental doses of sodium pentobarbital were administered intravenously, as it was necessary to maintain an adequate depth of anesthesia. The left kidney was exposed through a retroperitoneal flank incision. Then, a catheter (tapered PE-50) was inserted into the renal pelvis via the ureter. Urine was allowed to flow freely via a ureteral catheter. RSNA was recorded from the left renal nerve branch as previously described. ${ }^{15}$ Briefly, the renal nerve was isolated near the aortic-renal arterial junction, and a teflon-coated stainless steel bipolar electrode was placed under the nerve with the aid of an operation microscope. The renal nerve and electrode were covered with silicone rubber (semicosil $932 \mathrm{~A}$ and B, WackerChemie, Munich, Germany) and allowed to harden. RSNA was rectified and integrated (reset every $1 \mathrm{~s}$ ). The baseline noise, determined when nerve activity was eliminated by elevation of blood pressure with phenylephrine, was subtracted from the integrated RSNA. For the quantification of RSNA, the height of the integrated nerve discharge was recorded for $30 \mathrm{~s}$ in each stimulation. Changes in nerve activity were expressed as percentages of resting spontaneous nerve activity at baseline, before any elevation of pelvic pressure.

\section{Implantation of the guide cannula for ICV infusion}

A guide cannula was implanted into the left cerebroventricular region. Rats were placed on a stereotaxic frame (Narishige Scientific Instruments, Tokyo, Japan) in the prone position. The skin overlying the midline of the skull was incised, and a small hole was drilled in the dorsal surface of the cranium $0.9 \mathrm{~mm}$ posterior to the bregma and $1.5 \mathrm{~mm}$ lateral to the midline. A 23-gauge stainless-steel guide cannula was lowered $2 \mathrm{~mm}$ vertically from the dura toward the lateral ventricle. The guide cannula was fixed to the skull with screws and hardened together with dental cement.

\section{Other surgical preparations}

Renal denervation was performed to remove all visible and most length nerves surrounding the renal artery to the left kidney. The uninephrectomy of the right kidney was performed 1 week before the experiment. After the completion of surgery, each rat was left alone for $1 \mathrm{~h}$, to allow stabilization of blood pressure, RSNA and urine flow.

\section{Experimental protocols}

Effect of increases in pelvic pressure on systemic hemodynamics and RSNA. Animals were divided into intact (WKY rats, $n=10$; SHRs, $n=8$ ), uninephrectomized (WKY rats, $n=8$; SHRs, $n=8$ ) and denervated (WKY rats, $n=6$; SHRs, $n=6$ ) groups. Systemic blood pressure, heart rate (HR) and RSNA were recorded continuously throughout the experiment. HR was determined using a cardiotachometer. Pelvic pressure was increased by elevating the open end of the ureteral catheter $(5-50 \mathrm{~cm}$; above the pelvis level) and maintained for $1 \mathrm{~min}$. The intervals between the elevations of pelvic pressure were $5 \mathrm{~min}$ (or more), to enable the hemodynamic parameters to return to baseline. Responses of blood pressure, HR and RSNA to the changes in pelvic pressure were recorded. In the renal denervation group, RSNA was not measured.

\section{Effect of ICV administration of CV-11974 on cardiovascular responses to the changes in pelvic pressure}

In this study, eight WKY rats and nine SHRs were used. Artificial cerebrospinal fluid (a-CSF, $2 \mu \mathrm{l}$; Artcereb, Otsuka Pharmaceutical, Tokushima, Japan) was injected into the lateral cerebroventricular region. After $30 \mathrm{~min}$ of ICV injection of a-CSF, pelvic pressure was elevated by 5,20 and $50 \mathrm{~cm}$ for $1 \mathrm{~min}$. The intervals between the elevations of pelvic pressure were $5 \mathrm{~min}$ (or more), to enable hemodynamic parameters to return to baseline. Responses of blood pressure, HR and RSNA to the changes in pelvic pressure were recorded as control data. Then, an Ang II AT1 receptor antagonist (CV-11974, 1 and $10 \mu \mathrm{g})$ was injected into the lateral cerebroventricular region. The doses of CV-11974 were chosen, based on a report indicating that ICV infusion of CV-11074 (1 $\mu \mathrm{g})$ abolished the hypertensive effect of ICV injection of $100 \mathrm{ng}$ Ang II in rats. ${ }^{16}$ Thereafter, blood pressure, HR and RSNA responses to elevations of pelvic pressure were measured again.

ICV injection of agents was performed using an injector needle (30 gauge stainless-steel), which extended $1.5 \mathrm{~mm}$ beyond the tip of the guide cannula. Each injection of fluid and drugs was administered over a period more than $1 \mathrm{~min}(2 \mu \mathrm{l}$ volume). At the end of each experiment, rats received an ICV injection of 5\% methylene blue solution to verify the position of ICV injection.

\section{Statistical analysis}

All values are expressed as the mean \pm s.e. Data were analyzed by paired or unpaired Student's $t$-test, or two-way analysis of variance for repeated measures with a post hoc comparison (Bonferroni). A $P$-value of less than 0.05 was considered statistically significant.

\section{RESULTS}

Effect of increases in pelvic pressure on blood pressure and RSNA Baseline mean blood pressure (MBP) and HR of WKY rats and SHRs were $104 \pm 2.4 \mathrm{~mm} \mathrm{Hg}$ and $304 \pm 3.5$ beats per min, and $154 \pm 2.2 \mathrm{~mm} \mathrm{Hg}$ and $350 \pm 6.6$ beats per min, respectively. Typical traces of pulsatile blood pressure, HR and RSNA of each strain in response to increases in pelvic pressure are displayed in Figure 1. In both WKY rats and SHRs, blood pressure and RSNA were rapidly increased immediately after pelvic pressure was elevated. Then, increases in blood pressure and RSNA declined to their respective baseline levels after the ureteral catheter was restored to its original position in both strains. As shown in Figure 2, the elevations of pelvic pressure significantly increased MBP and RSNA of WKY rats and SHRs in a pressure-dependent fashion. Similar changes were observed in contralateral nephrectomized WKY rats and SHRs (Figure 2). However, in WKY rats and SHRs subjected to renal denervation, the responses of MBP were eliminated (Figure 2). On the other hand, HR responses to increasing pelvic pressure were not consistent across the animals and the results were highly variable; thus, analysis of HR was not performed in the following experiments.

\section{Effect of ICV injection of CV-11974 on blood pressure and RSNA} responses to changes in pelvic pressure

Administration of a-CSF had no effect on any baseline parameters in both strains of rats (Table 1). Furthermore, a-CSF treatment did not affect the elevations of MBP and RSNA of either strain in response to increased pelvic pressure (Figures 2, 3 and 4). ICV injection of 


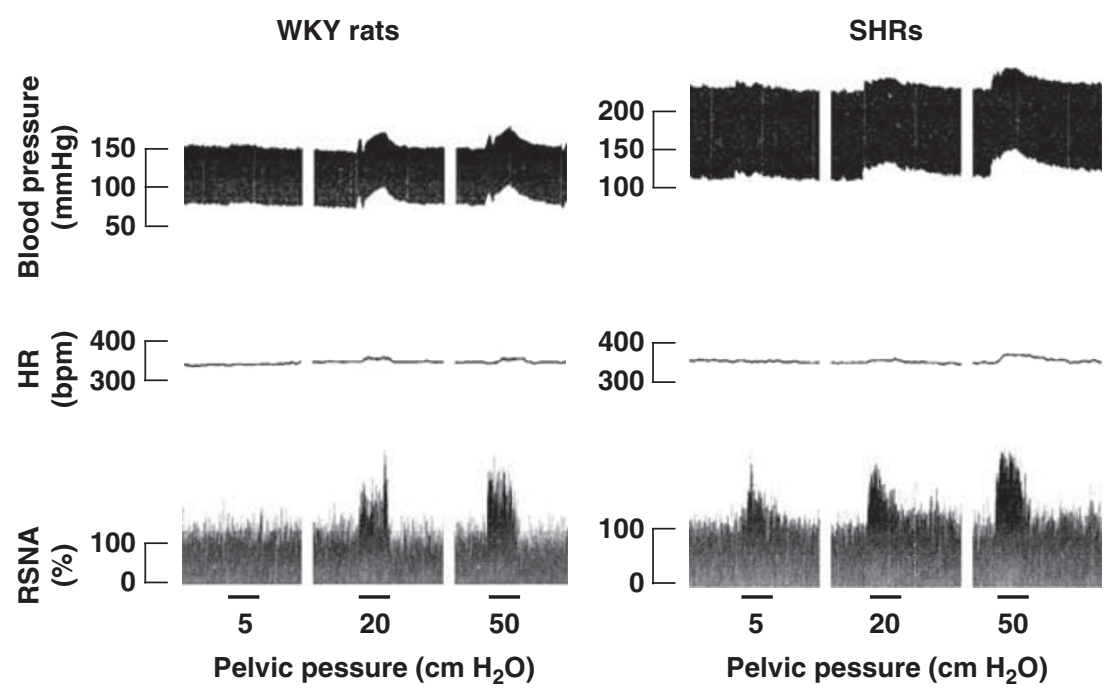

Figure 1 Typical trace of pulsatile blood pressure, heart rate (HR) and renal sympathetic nerve activity (RSNA) in response to increases in renal pelvic pressure in Wistar-kyoto Izm (WKY) rat and spontaneously hypertensive Izm rat (SHR).
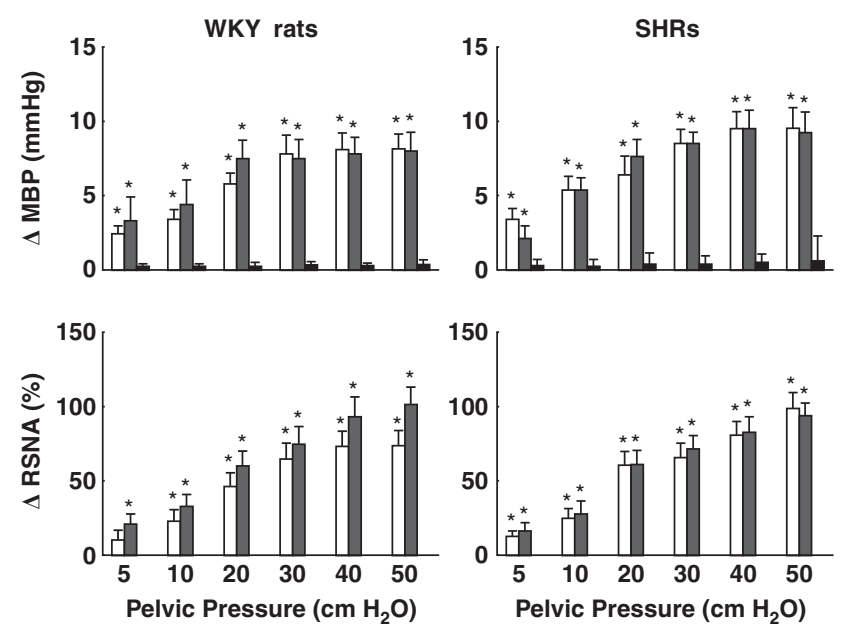

Figure 2 Effect of increasing renal pelvic pressure on mean blood pressure (MBP) and renal sympathetic nerve activity (RSNA) in Wistar-Kyoto Izm (WKY) rats and spontaneously hypertensive Izm rats (SHRs). Open column, intact; dotted column, contralateral nephrectomy; filled column, denervation. ${ }^{*} P<0.05$ vs. control.

CV-11974 (1 or $10 \mu \mathrm{g})$ significantly decreased baseline MBP in SHRs, but not in WKY rats (Table 1). Similarly, ICV injection of $10 \mu \mathrm{g} \mathrm{CV-}$ 11974 significantly increased baseline RSNA in SHRs, but not in WKY rats, whereas ICV injection of $1 \mu \mathrm{g} \mathrm{CV-11974} \mathrm{did} \mathrm{not} \mathrm{change} \mathrm{baseline}$ RSNA in either strain. ICV injection of CV-11974 $(1 \mu \mathrm{g})$ tended to suppress the MBP and RSNA responses to increased pelvic pressure in both strains, but these effects were not statistically significant when compared with those in respective a-CSF-treated rats. On the other hand, CV-11974 $(10 \mu \mathrm{g})$ significantly suppressed the vasopressor and sympathoexcitative responses of both strains with increased pelvic pressure at $5 \mathrm{~cm} \mathrm{H}_{2} \mathrm{O}$, when compared with control (a-CSF treated) rats. The inhibitory effect of CV-11974 appeared to be greater in WKY rats than SHRs (Figures 3 and 4). The vasopressor and sympathoexcitative responses in WKY rats at 20 and $50 \mathrm{~cm} \mathrm{H}_{2} \mathrm{O}$ pelvic pressures were significantly attenuated, compared with the control group.
Table 1 Effect of intracerebroventricular injection of a-CSF and CV-11974 on MBP, HR and RSNA in WKY rats and SHRs

\begin{tabular}{lcll}
\hline & $M B P(\mathrm{~mm} \mathrm{Hg})$ & $H R($ b.p.m. $)$ & RSNA (\%) \\
\hline WKY rats $(n=8)$ & & & \\
$\quad$ No treatment & $106 \pm 3$ & $335 \pm 5$ & $100 \pm 8$ \\
a-CSF $(2 \mu \mathrm{l})$ & $108 \pm 2$ & $337 \pm 6$ & $105 \pm 4$ \\
CV-11974 $(1 \mu \mathrm{g})$ & $107 \pm 2$ & $342 \pm 12$ & $110 \pm 9$ \\
$(10 \mu \mathrm{g})$ & $106 \pm 3$ & $363 \pm 11$ & $115 \pm 11$ \\
& & & \\
SHRs $(n=9)$ & & & \\
No treatment & $150 \pm 3$ & $352 \pm 5$ & $100 \pm 7$ \\
a-CSF $(2 \mu \mathrm{l})$ & $146 \pm 2$ & $349 \pm 6$ & $102 \pm 5$ \\
CV-11974 $(1 \mu \mathrm{g})$ & $140 \pm 2^{*}$ & $358 \pm 6$ & $110 \pm 8$ \\
$(10 \mu \mathrm{g})$ & $132 \pm 2^{*}$ & $374 \pm 8$ & $120 \pm 9 *$ \\
\hline
\end{tabular}

Abbreviations: a-CSF, artificial cerebrospinal fluid; HR, heart rate; MBP, mean blood pressure; RSNA, renal sympathetic nerve activity; SHRs, spontaneously hypertensive Izm rats; WKY, Wistar-Kyoto Izm rat.

Values are mean \pm s.e.

${ }^{*} P<0.05$ vs. no treatment.

\section{DISCUSSION}

Recently, Schlaich et al. ${ }^{7}$ have shown that renal denervation by mechanical nerve ablation significantly decreases blood pressure in uncontrolled hypertensive patients. However, the mechanisms by which renal denervation decreases blood pressure have remained uncertain. The present study demonstrated that activation of ARNs by pelvic pressure elevation caused an increase in both RSNA and blood pressure. Furthermore, ICV injection of an AT1 receptor antagonist significantly attenuated the increase in RSNA and blood pressure in response to elevated pelvic pressure. To the best of our knowledge, these data indicate, for the first time, that ARNs have an important role in the control of systemic autonomic and hemodynamic systems through regulating the CNS RAS-dependent pathways. Our data also suggest the possibility that renal denervation by mechanical nerve ablation decreases blood pressure by inhibiting these pathways.

It is well known that ARNs convey information from the kidney to the CNS. ${ }^{17,18}$ In the present study, we observed that elevations of pelvic pressure increased RSNA and blood pressure. Furthermore, 

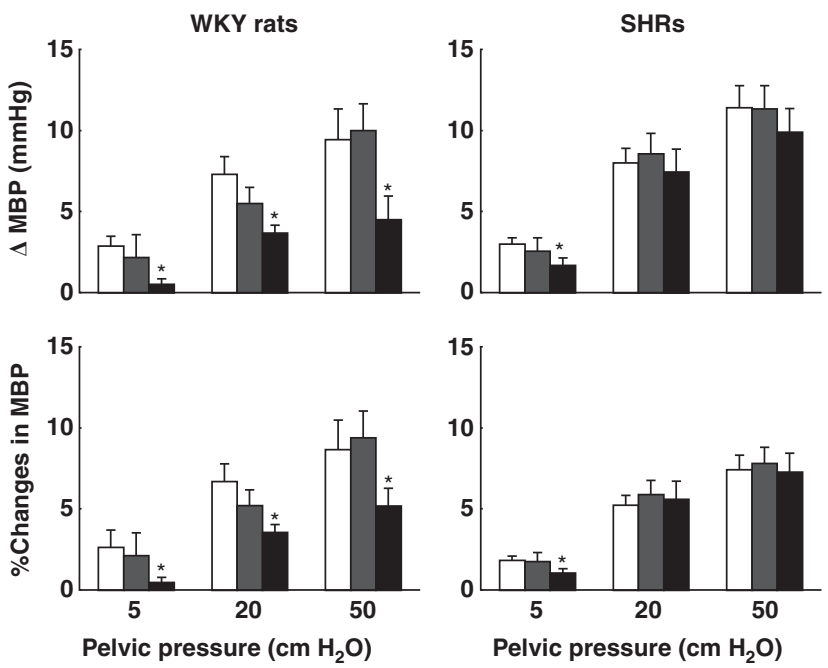

Figure 3 Effect of intracerebroventricular (ICV) injection of CV-11974 on mean blood pressure (MBP) responses to changes in renal pelvic pressure in Wistar-Kyoto Izm (WKY) rats and spontaneously hypertensive Izm rats (SHRs). Upper panel: actual measured values of changes in MBP. Lower panel: percent changes of baseline value in MBP. Open column, artificial cerebrospinal fluid (a-CSF); dotted column, CV-11974 (1 $\mu \mathrm{g})$; filled column,

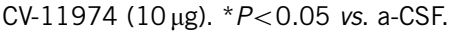
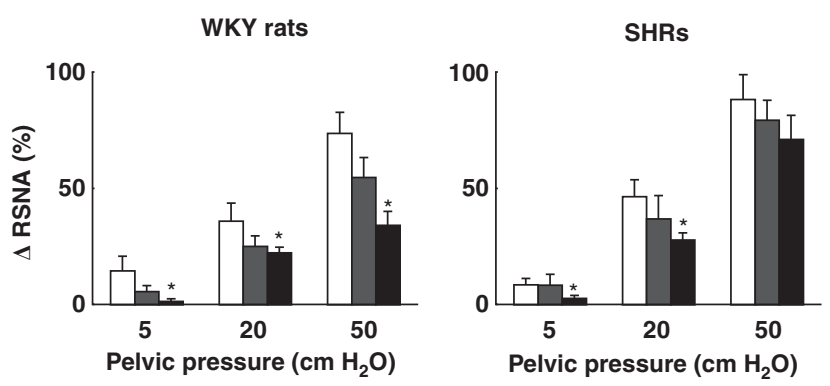

Figure 4 Effect of intracerebroventricular (ICV) injection of CV-11974 on renal sympathetic nerve activity (RSNA) responses to changes in renal pelvic pressure in Wistar-Kyoto Izm (WKY) rats and spontaneously hypertensive Izm rats (SHRs). Open column, artificial cerebrospinal fluid (a-CSF); dotted

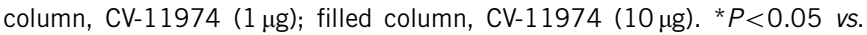
a-CSF.

increased blood pressure was abolished by renal denervation. These data suggest that the activation of ARNs induced by the alteration in the renal circumstances, that is, increased renal pelvic pressure, caused systemic sympathetic activation and vasopressor response. Overactivity in the sympathetic nervous system is observed in patients with renal disease. ${ }^{19}$ Furthermore, nephrectomy or renal denervation has been shown to correct blood pressure and sympathetic nerve activity. ${ }^{6,7,20}$ Collectively, these data are consistent with the concept that the increase in renal afferent inputs to the CNS from the diseased kidney, leading to systemic sympathetic nerve overactivity and the development of hypertension in patients with chronic kidney disease.

Central Ang II has been implicated as a factor for the development and maintenance of hypertension. ${ }^{10,21}$ The cardiovascular and autonomic systems are regulated by AT1 receptors expressed in the CNS, including the hypothalamus, the nucleus tractus solitarius and the rostral ventrolateral medulla. ${ }^{22,23}$ Brain Ang II activates peripheral sympathetic nerve activity via arterial baroreceptor gain and activation of preganglionic neurons. ${ }^{21,24}$ Changes in blood pressure, as well as RSNA and norepinephrine secretion from the posterior hypothalamic nuclei that are induced by an intrarenal injection of phenol, can all be inhibited by ICV infusion of an AT1 receptor antagonist, losartan. ${ }^{25}$ Furthermore, central Ang II enhances RSNA reflex increase by cardiac afferent stimulation. ${ }^{9}$ It has also been reported that ARNs are connected to the central areas, including the nucleus tractus solitarius, the paraventricular nucleus, the supraoptic nucleus, the subfornical organ and others. ${ }^{26,27}$ In the present study, ICV injection of an Ang II AT1 receptor antagonist, CV-11974, significantly attenuated the increases in RSNA and blood pressure in response to elevation of pelvic pressure. Collectively, these findings support the hypothesis that afferent nervous information from the kidney is transmitted to the CNS and stimulates the systemic sympathetic activity via the CNS RAS-mediated pathways.

The expression of AT1 receptors in the central areas of SHRs is higher than that of WKY rats. ${ }^{10,28}$ In the present study, ICV administration of an AT1 receptor antagonist, CV-11974 (10 $\mu \mathrm{g})$, significantly attenuated the vasopressor and sympathoexcitatory responses to increased pelvic pressure in WKY rats. Similar effects were observed in SHRs as well, but these effects of CV-11974 appeared to be weaker in SHRs than in WKY rats. These data suggest that in SHRs, Ang II does not have a predominant role in excitatory regulation of the sympathetic nervous system, following the transmission of renal afferent signaling into the CNS. Ito et al. ${ }^{29}$ showed that bilateral injection of kynurenic acid, an excitatory amino acid antagonist, into the rostral ventrolateral medulla did not alter blood pressure in WKY rats, whereas it significantly reduced blood pressure in SHRs. Similarly, inhibition of glutamatergic inputs in the paraventricular nucleus of the hypothalamus decreased blood pressure and sympathetic outflow in SHRs, but not in WKY rats. ${ }^{30}$ These data suggest that, during the development of hypertension in SHRs, an excitatory influence is exerted on the sympathetic regulation in the CNS through the glutamatergic pathways. Therefore, ARN-induced sympathoexcitatory responses may also be caused by some factors other than CNS RAS, for example, glutamatergic pathways in SHRs.

Our data suggest that the CNS RAS has an important role in regulating cardiovascular hemodynamics in response to the activation of ARNs. However, a weak point of the present study is that we cannot address any issue regarding the actual functional site(s) of the CNS RAS in the brain. Previous studies have shown that ICV administration of CV-11974 at $1 \mu \mathrm{g}$ inhibits the increase in blood pressure induced by exogenous ICV injection of AngII in rats. ${ }^{16}$ In agreement with these data, our data also showed that ICV administration of $1 \mu \mathrm{g}$ CV-11974 had a tendency to suppress the RSNA responses to increased pelvic pressure. However, as these effects of CV-11974 were not statistically significant, we speculate that CNS RAS is involved in the control of the sympathetic nervous system, predominantly at certain sites in brain parenchyma, such as paraventricular nucleus, that are not easily accessed by the exogenous CV-11974 (1 $\mu \mathrm{g})$ administered into cerebroventricular regions. It is also possible that CSN Ang II levels are changed in response to increases in pelvic pressure. However, we failed to measure the Ang II levels in cerebroventricular regions due to the technical difficulties. Further studies are required to address these issues.

Previous studies have reported that similar stimulation to the kidney activated ARN activity, but did not show any significant change in blood pressure due to renorenal reflex function. ${ }^{11-14,31}$ The reason for the discrepancy between our result and other studies is not clear. However, the present study also showed that blood pressure and RSNA were consistently increased in response to elevated 
pelvic pressure in the contralaterally nephrectomized rats. Thus, increases in blood pressure and RSNA induced by the activation of ARNs appear to have occurred under the present experimental conditions, where renorenal reflex function is not present.

In conclusion, the present study supports the hypothesis that ARN activity controls cardiovascular hemodynamics by regulating systemic sympathetic activity through the CNS RAS. Our results also suggest that these pathways can be a potential target for the treatment of hypertension accompanied by hyperactivity of the ARNs in patients with chronic kidney disease.

\section{CONFLICT OF INTEREST}

The authors declare no conflict of interest.

1 Ciriello J. Afferent renal inputs to paraventricular nucleus vasopressin and oxytocin neurosecretory neurons. Am J Physiol 1998; 275: R1745-R1754.

2 Caverson MM, Ciriello J. Effect of stimulation of afferent renal nerves on plasma levels of vasopressin. Am J Physiol 1987; 252: R801-R807.

3 Smits JF, Brody MJ. Activation of afferent renal nerves by intrarenal bradykinin in conscious rats. Am J Physiol 1984; 247: R1003-R1008.

4 Katholi RE, Hageman GR, Whitlow PL, Woods WT. Hemodynamic and afferent renal nerve responses to intrarenal adenosine in the dog. Hypertension 1983; 5: I149-I154.

5 Ye S, Zhong H, Yanamadala V, Campese VM. Renal injury caused by intrarenal injection of phenol increases afferent and efferent renal sympathetic nerve activity. Am J Hypertens 2002; 15: 717-724.

6 Converse Jr RL, Jacobsen TN, Toto RD, Jost CM, Cosentino F, Fouad-Tarazi F, Victor RG. Sympathetic overactivity in patients with chronic renal failure. N Engl J Med 1992; 327: 1912-1918.

7 Schlaich MP, Sobotka PA, Krum H, Lambert E, Esler MD. Renal sympathetic-nerve ablation for uncontrolled hypertension. N Engl J Med 2009; 361: 932-934.

8 Campese VM, Ye S, Zhong $\mathrm{H}$. Downregulation of neuronal nitric oxide synthase and interleukin-1 beta mediates angiotensin II-dependent stimulation of sympathetic nerve activity. Hypertension 2002; 39: 519-524.

9 Zhu GQ, Patel KP, Zucker IH, Wang W. Microinjection of ANG II into paraventricular nucleus enhances cardiac sympathetic afferent reflex in rats. Am J Physiol Heart Circ Physiol 2002; 282: H2039-H2045.

10 Ito S, Komatsu K, Tsukamoto K, Kanmatsuse K, Sved AF. Ventrolateral medulla AT1 receptors support blood pressure in hypertensive rats. Hypertension 2002; 40: 552-559.

11 Kopp UC, Cicha MZ, Farley DM, Smith LA, Dixon BS. Renal substance P-containing neurons and substance $P$ receptors impaired in hypertension. Hypertension 1998; 31: 815-822.
12 Kopp UC, Cicha MZ, Smith LA. Impaired responsiveness of renal mechanosensory nerves in heart failure: role of endogenous angiotensin. Am J Physiol Regul Integr Comp Physiol 2003; 284: R116-R124.

13 Recordati GM, Moss NG, Genovesi S, Rogenes PR. Renal receptors in the rat sensitive to chemical alterations of their environment. Circ Res 1980; 46: 395-405.

14 Recordati G, Genovesi S, Cerati D. Renorenal reflexes in the rat elicited upon stimulation of renal chemoreceptors. J Auton Nerv Syst 1982; 6: 127-142.

15 Fujisawa Y, Mori N, Yube K, Miyanaka H, Miyatake A, Abe Y. Role of nitric oxide in regulation of renal sympathetic nerve activity during hemorrhage in conscious rats. Am J Physiol 1999; 277: H8-14.

16 Bunting MW, Widdop RE. Lack of a centrally-mediated antihypertensive effect following acute or chronic central treatment with AT1-receptor antagonists in spontaneously hypertensive rats. Br J Pharmacol 1995; 116: 3181-3190.

17 Calaresu FR, Ciriello J. Renal afferent nerves affect discharge rate of medullary and hypothalamic single units in the cat. J Auton Nerv Syst 1981; 3: 311-320.

18 Ciriello J, Calaresu FR. Central projections of afferent renal fibers in the rat: an anterograde transport study of horseradish peroxidase. J Auton Nerv Syst 1983; 8: 273-285.

19 Phillips JK. Pathogenesis of hypertension in renal failure: role of the sympathetic nervous system and renal afferents. Clin Exp Pharmacol Physiol 2005; 32: 415-418.

20 Schlaich MP, Sobotka PA, Krum H, Whitbourn R, Walton A, Esler MD. Renal denervation as a therapeutic approach for hypertension: novel implications for an old concept. Hypertension 2009; 54: 1195-1201.

21 DiBona GF, Jones SY, Sawin LL. Effect of endogenous angiotensin II on renal nerve activity and its arterial baroreflex regulation. Am J Physiol 1996; 271: R361-R367.

22 Gehlert DR, Gackenheimer SL, Schober DA. Autoradiographic localization of subtypes of angiotensin II antagonist binding in the rat brain. Neuroscience 1991; 44: 501-514.

23 Tsutsumi K, Saavedra JM. Characterization and development of angiotensin II receptor subtypes (AT1 and AT2) in rat brain. Am J Physiol 1991; 261: R209-R216.

24 Sasaki S, Dampney RA. Tonic cardiovascular effects of angiotensin II in the ventrolateral medulla. Hypertension 1990; 15: 274-283.

25 Ye S, Zhong H, Duong VN, Campese VM. Losartan reduces central and peripheral sympathetic nerve activity in a rat model of neurogenic hypertension. Hypertension 2002; 39: 1101-1106.

26 Moncrief K, Hamza S, Kaufman S. Splenic reflex modulation of central cardiovascular regulatory pathways. Am J Physiol Regul Integr Comp Physiol 2007; 293: R234-R242.

27 Nishimura M. Takahashi H, Yoshimura M. Upregulation of the brain renin-angiotensin system in rats with chronic renal failure. Acta Physiol (Oxf) 2007; 189: 369-377.

28 Koga Y, Hirooka Y, Araki S, Nozoe M, Kishi T, Sunagawa K. High salt intake enhances blood pressure increase during development of hypertension via oxidative stress in rostral ventrolateral medulla of spontaneously hypertensive rats. Hypertens Res 2008; 31: 2075-2083.

29 Ito S, Komatsu K, Tsukamoto K, Sved AF. Excitatory amino acids in the rostral ventrolateral medulla support blood pressure in spontaneously hypertensive rats. Hypertension 2000; 35: 413-417.

$30 \mathrm{Li}$ DP, Pan HL. Glutamatergic inputs in the hypothalamic paraventricular nucleus maintain sympathetic vasomotor tone in hypertension. Hypertension 2007; 49: 916-925.

31 Genovesi S, Pieruzzi F, Camisasca P, Golin R, Zanchetti A, Stella A. Renal afferents responsive to chemical and mechanical pelvic stimuli in the rabbit. Clin Sci (Lond) 1997; 92: 505-510. 